\title{
Exposición ocupacional y cáncer de pulmón en fumadores
}

\author{
Occupational exposure and lung cancer in smokers
}

\begin{abstract}
Ricardo Mahuad*, Stella Pezotto**, Leonor Poletto**
MAHUAD, R. et al. Exposición ocupacional y cáncer de pulmón en fumadores. Rev. Saúde Pública, 28: 204-8, 1994. Las altas tasas de incidencia y mortalidad por cáncer de pulmón en hombres en Rosario, Argentina motivaron o estudio de 211 pacientes varones. Se comparon las edades al diagnóstico entre los tipos histológicos y ocupaciones, y edad de iniciación, duración e intensidad del hábito de fumar. La edad promedio al diagnóstico fue 61 años, sin diferencias por tipo histológico pero sí por hábito de fumar: 58 años los fumadores y 68 los ex-fumadores $(p<0,0001)$. En estos pacientes se encontró un predominio de tumores epidermoides, lo que podria estar asociado a los contaminantes ocupacionales presentes en esta etapa de industrialización. La asociación entre tipo cèlular y ocupación fue altamente significativa ( $<<0,0001$ ), encontrándose el adenocarcinoma con mayor frecuencia en administrativos (oficinistas, docentes, contadores, abogados) y el epidermoide en otras ocupaciones (agricultores, metalúrgicos), especialmente en quienes fueron primeramente agricultores y luego metalúrgicos.
\end{abstract}

Descriptores: Neoplasmas pulmonares, epidemiología. Tabaquismo. Ocupaciones.

\section{Introducción}

Es necesario considerar primeramente la magnitud de la incidencia y mortalidad por cáncer de pulmón en Argentina. En la Investigación Interamericana de Mortalidad $^{18}$, La Plata, ciudad argentina participante, presentó la tasa de mortalidad ajustada por edad, en el hombre, mas alta del continente $(59 \% 000)$. En un trabajo posterior realizado en Rosario, ésta fue de $65 \% 000^{15}$. Según la información proporcionada por el Registro de Tumores de esta ciudad, el que se encuentra en etapa organizativa, su tasa de incidencia fue de $61 \% 000$ en 1988. Tanto la breve sobrevida como el reducido número de curaciones apoyan la coherencia de estas tasas.

La asociación etiológica entre el hábito de fumar cigarrillos y el cáncer primitivo de pulmón ha sido firmemente demostrada en las últimas décadas ${ }^{21}$. En cambio, las exposiciones ocupacionales, así como los factores determinantes de tipos histológicos específi$\cos$, se encuentran poco caracterizados ${ }^{1,27}$. En una revisión de estudios americanos y europeos, en los que se había controlado el hábito de fumar como factor de confusión, se observó que la proporción de estos

\footnotetext{
* Servicio de Neumonologia, Hospital Italiano, Rosario, Argentina.

** Instituto de Inmunologia. Facultad de Ciencias Médicas. Universidad Nacional de Rosario, Rosario, Argentina Separatas/Reprints: L.Poletto - Santa Fé 3.100-2.000 Rosario, Argentina
}

tumores debidos a exposiciones ocupacionales vario entre 0 y $40 \%{ }^{20}$.

Los estudios clínico-epidemiológicos referidos a las características de las asociaciones de factores de riesgo de cáncer de pulmón son escasos en Latinoamérica. La gran mayoría de los trabajos sobre riesgos ocupacionales procede del hemisferio norte, pero sus resultados no son fácilmente extrapolables a los ambientes laborales de los países en desarrollo.

En el presente trabajo se propuso evaluar las exposiciones ocupacionales así como la duración e intensidad del hábito de fumar como determinantes de los tipos histológicos en pacientes con cáncer primitivo de pulmón.

\section{Material y Metodo}

Se estudiaron pacientes que concurrieron espontáneamente a la consulta médica a dos instituciones asistenciales de la ciudad de Rosario durante 1987 1991 y recibieron el diagnóstico histopatológico de cáncer pulmonar primitivo.

Se diagnosticaron 233 pacientes, 215 hombres y 18 mujeres. Se excluyeron de los análisis 4 hombres no fumadores ( $2 \%$ ), así como a las mujeres, dada la alta proporción (16/18) de amas de casa como actividad ocupacional. De éstas, 8 eran fumadoras (44\%) y 5 fumadoras pasivas. Se incorporaron al estudio 211 pacientes masculinos, 165 fumadores (77\%) y 46 exfumadores $(21 \%)$. Todos eran fumadores de cigarrillos. 
Los pacientes fueron estudiados a través de broncoscopía con biopsia. Los diagnósticos fueron hechos por el mismo equipo de anatomopatólogos y todos fueron revisados por el Jefe de Sección, utilizándose la clasificación de la OMS 1981. Se constituyeron cuatro grupos según los tipos celulares: adenocarcinoma, epidermoide, células pequeñas y 'otros' tipos histológicos incluyendo a aquellos sin especificar.

Se documentó la historia ocupacional de cada paciente sobre denominación y características de su trabajo y tipo de industria y/o empresa, referida a cada una de las ocupaciones desarrolladas durante mas de un año. Se prefirió este sistema de clasificación de trabajos al de las matrices de exposición por ocupaciones por considerar que éstas dan mayores estimaciones de riesgo ${ }^{28}$. Las ocupaciones fueron codificadas acorde con la Clasificación Internacional Uniforme de Ocupaciones ${ }^{6}$, la que fue adaptada a las características laborales locales. Las características ocupacionales de estos pacientes decidieron la formación de los siguientes grupos: 1- 'administrativos', incluyendo a todas las actividades en las que no habría exposiciones a contaminantes, como ser oficinistas, telefónicos, docentes, abogados, contadores; 2- 'metalúrgicos', trabajadores de la fundición, laminación, torneros, mecánicos; 3- 'agricultores', involucrando a todas las actividades agropecuarias en las que se puede tomar contacto con agroquímicos; 4- 'agricultoresmetalúrgicos', comprende a aquellos hombres que iniciaron su vida laboral en actividades del campo y que luego se trasladaron a la ciudad incorporándose a tareas metalúrgicas; 5- en 'otras' ocupaciones se incluyó a carpinteros, albañiles y choferes por sus reducidos números.

Los pacientes fueron agrupados en tres categorías acorde con el hábito de fumar: no fumadores, ex-fumadores y fumadores. Se registró la edad de iniciación y abandono del hábito, su intensidad y duración. Además se indagó sobre su condición e intensidad de fumadores pasivos.

El análisis estadístico consistió en descripción de frecuencias de las variables estudiadas, análisis de las asociaciones de las mismas mediante la prueba 'chi' cuadrado y análisis de la variancia para la comparación de promedios.

\section{Resultados}

Se encontró la siguiente distribución de los pacientes por tipos histológicos: epidermoides $39 \%$, adenocarcinomas $29 \%$, células pequeñas $18 \%$ y otros tipos celulares y no especificados $14 \%$.

La clasificación por ocupaciones según el tipo histologico se muestra en Tabla 1. La asociación entre tipo celular y ocupación es altamente significativa $(\mathrm{p}<0,001)$ al considerar los tipos histologicos y las ocupaciones mas prevalentes. El adenocarcinoma se present 6 con mas frecuencia en los administrativos y el epidermoide en las otras ocupaciones, especialmente en quienes fueron primeramente agricultores y luego metalúrgicos.

Al analizarse la edad promedio al diagnóstico por

Tabla1. Tipos histológicos según ocupación

\begin{tabular}{lccccc}
\hline \multirow{2}{*}{ Ocupación } & \multicolumn{3}{c}{ Tipos histológicos } & Total \\
\cline { 2 - 5 } & Epidermoide & Adenocarcinoma & Células pequeñas & Otros \\
\hline Administrativos & 11 & 28 & 13 & 10 & 62 \\
Metalúrgicos & 20 & 11 & 6 & 9 & 46 \\
Agricultores & 20 & 8 & 8 & 4 & 40 \\
Agric-Metal. & 23 & 7 & 3 & 2 & 35 \\
Otras & 11 & 6 & 6 & 5 & 28 \\
\hline Total & 85 & 60 & 36 & 30 & 211 \\
\hline
\end{tabular}

$X^{2}=24,44 ; 6$ grados de libertad (excluyendo otros tipos histológicos y otras ocupaciones); $p<0,001$.

Tabla 2. Edad promedio al diagnóstico según tipo histológico y ocupación

\begin{tabular}{lccc}
\hline Ocupación & \multicolumn{3}{c}{ Tipos histológicos } \\
\cline { 2 - 4 } & Epidermoide & Adenocarcinoma & Células pequeñas* $^{*}$ \\
\hline Administrativos & $61 \pm 3,4$ & $62 \pm 1,9$ & $62 \pm 1,6$ \\
Metalúrgicos & $56 \pm 1,6$ & $59 \pm 2,8$ & $49 \pm 2,5$ \\
Agricultores & $62 \pm 2,8$ & $64 \pm 2,6$ & $60 \pm 2,9$ \\
Agric-Metal. & $61 \pm 1,4$ & $59 \pm 3,0$ & $60 \pm 3,1$ \\
\hline
\end{tabular}

* $p=0,02$. 
Tabla 3. Intensidad del hábito de fumar según ocupación

\begin{tabular}{lcccc}
\hline Ocupación & \multicolumn{2}{c}{ Intensidad ${ }^{*}$} & Total \\
\cline { 2 - 5 } & I & II & III & 62 \\
\hline Administrativos & 9 & 23 & 30 & 46 \\
Metalúrgicos & 5 & 24 & 17 & 40 \\
Agricultores & 2 & 17 & 15 & 35 \\
Agric-Metal. & 3 & 17 & 83 & 183 \\
\hline Total & 19 & 81 & 15 & \\
\hline
\end{tabular}

* Intensidad del hábito:

l: $\leq 20 \mathrm{cig} /$ día $y \leq 40$ años fumando;

II: $\leq 20 \mathrm{cig} / \mathrm{di} a \mathrm{y}$ y $>40$ años $0>20 \mathrm{cig} / \mathrm{dí}$ y $\leq 40$ años;

III: $>20$ cig/día y $>40$ años fumando.

Tabla 4. Edad promedio de iniciación al hábito de fumar según tipo histológico

\begin{tabular}{lccc}
\hline Hábito & \multicolumn{3}{c}{ Tipos histológicos } \\
\cline { 2 - 4 } & Epidermoide & Adenocarcinoma & Células pequeñas \\
\hline Fumador & $14,6 \pm 0,4$ & $17,2 \pm 0,7$ & $14,9 \pm 0,6$ \\
Ex-Fumador & $14,9 \pm 0,6$ & $16,5 \pm 0,8$ & $16,0 \pm 0,9$ \\
\hline Total & $14,6 \pm 0,4$ & $17,0 \pm 0,7$ & $15,1 \pm 0,6$ \\
\hline
\end{tabular}

Diferencias por tipos histológicos: p<0,001; hábito de fumar: NS.

Tabla 5. Edad promedio al diagnóstico según tipo histológico y hábito de fumar

\begin{tabular}{lccc}
\hline Hábito & \multicolumn{3}{c}{ Tipos histológicos } \\
\cline { 2 - 4 } & Epidermoide & Adenocarcinoma & Células pequeñas $^{*}$ \\
\hline Fumador & $59,0 \pm 1,0$ & $58,7 \pm 1,3$ & $57,3 \pm 1,6$ \\
Ex-fumador & $68,5 \pm 1,7$ & $68,8 \pm 1,2$ & $69,5 \pm 2,9$ \\
\hline Total & $60,9 \pm 1,0$ & $60,9 \pm 1,2$ & $59,3 \pm 1,7$ \\
\hline
\end{tabular}

Diferencias por tipos histológicos: NS; hábito de fumar: $p<0,0001$.

ocupaciones (Tabla 2) se observa la menor edad de los trabajadores metalúrgicos en los tres tipos histológi$c o s$, siendo significativa su diferencia en los epidermoides y células pequeñas $(\mathrm{p}=0,02)$.

En la Tabla 3 se muestra la distribución de los pacientes en las ocupaciones mas prevalentes $(\mathbf{n}=183)$ según la asociación de la intensidad y duración del hábito. No se encontraron diferencias en el consumo de cigarrillos por ocupaciones $\left(X^{2}=5,20, N S\right)$.

Al considerar la edad de iniciación en el hábito de fumar (Tabla 4) se observó que ésta fue significativamente mas tardía en los pacientes que desarrollaron adenocarcinoma $(p<0,001)$. Pero no se encontraron diferencias al analizar para cada tipo histológico la condición de fumador o ex-fumador.

En cambio, se encontraron diferencias altamente significativas $(\mathrm{p}<0,0001)$ entre las condiciones de fumador y ex-fumador al analizar la edad al diagnóstico de estos pacientes, para cada tipo histológico (Tabla 5). Los ex-fumadores fueron 10 años mayores que los fumadores en los tres tipos histológicos mas prevalentes. No se encontraron diferencias entre las edades de los pacientes por tipo celular en los fumadores ni en los ex-fumadores.

Además, debe tenerse en cuenta que el promedio de los años de abandono del hábito (13 años) en los exfumadores, tampoco fue significativo en su asociación con el diagnóstico histopatológico. A su vez, la duración del hábito, promedio 42 años, no presentó diferencias entre fumadores y ex-fumadores, ni entre tipos celulares.

\section{Discusión}

Si bien el hábito de fumar cigarrillos es la principal causa reconocida de cáncer de pulmón en todo el mundo, en diversos trabajos se han observado importantes variaciones geográficas en su incidencia, las que persistieron luego de controlar la edad y el consumo 
de cigarrillos ${ }^{13}$. Cuando un factor de riesgo, de consumo masivo como ocurre con el cigarrillo, eleva la tasa basal de incidencia de una enfermedad, una contribución aditiva producida por otro y otros factores resulta difícil de detectar por transformarse en relativamente marginal ${ }^{2}$. La alta ingesta de grasas así como la reducida participación de frutas y vegetales en la dieta han sido estudiados como factores de riesgo, pero con resultados no coincidentes ${ }^{3,29}$. Al investigarse la participación de la residencia urbana o rural, considerando la mayor contaminación medioambiental en las ciuda$\mathrm{des}^{26}$, sí bien se observaron asociaciones positivas con la urbanización éstas no fueron concluyentes. Es posible que estos resultados no definitorios sean producidos por la falta de control de la variable ocupacional.

$\mathrm{La}$ asociación negativa encontrada en países desarrollados entre la incidencia de cáncer de pulmón y las clases sociales ${ }^{9}$ no se reiteró en Rosario ${ }^{16}$, donde la asociación fue positiva, al igual que en un estudio brasileñ ${ }^{7}$. Esto es concordante con ciertas características de esta problación, en la que, a la inversa de lo que se ha dado en países desarrollados ${ }^{19}$, persiste la mayor prevalencia de fumadores en los estratos socioeconomicos mas altos ${ }^{17}$. En éstos, a pesar de estar menos expuestos a contaminantes ocupacionales, el tabaquismo sería el responsable de las mayores tasas de incidencia que presentaron.

La edad promedio al diagnóstico de los pacientes ex-fumadores, en los tres tipos histológicos mas prevalentes, fue significativamente mayor en 10 años que en los fumadores, característica no hallada en la bibliografía consultada.

Otro hecho distintivo a señalar es la significativa diferencia en la edad de iniciación en el hábito de fumar de estos pacientes, según tipos celulares, siendo aquellos con adenocarcinoma quienes mas tardiamente lo hicieron.

A diferencia de lo observado en otras poblaciones, en las que se habría dado un incremento y actual predominio de los adenocarcinomas ${ }^{12,25}$, en este trabajo se encontró una mayor proporción de epidermoides, lo que es coincidente con el estudio histopatológico del cáncer realizado en Brasil ${ }^{4}$, y estaría en consonancia con lo que se verificó en algunos países en décadas pasadas ${ }^{3}$.

Kreyberg ${ }^{8}$ propuso una controvertida clasificación de este tumor en dos grupos, fundamentada en sus características epidemiológicas. El grupo 1 comprende a los tumores que se desarrollan principalmente en las porciones centrales del tracto respiratorio, epidermoides y células pequeñas. Los del grupo 2 son periféricos y fundamentalmente adenocarcinomas. Los tipos celulares del grupo 1 serían los mas asociados a carcinógenos inhalados, como el humo del cigarrillo.

La importancia de la participación de los contaminantes ocupacionales en la cadena etiológica de este tumor está siendo reconocida ${ }^{11,20,23,24}$. El acentuado predominio de los tumores epidermoides en USA y otros países hasta hace algunos años y su desplazamiento por el incremento de los adenocarcinomas, en ambos sexos, podría estar asociado a los cambios ocurridos en los factores contaminantes medioambientales ocupacionales. En este estudio se encontró una mayor prevalencia de adenocarcinoma en administrativos (42\%), aparentemente no expuestos a contaminantes ocupacionales, pero sí a los elementos químicos del humo del cigarrillo, puesto que todos estos pacientes eran fumadores, lo que estaría en contradicción con lo señalado por Kreyberg8. Los tumores epidermoides prevalecieron en los agricultores, metalúrgicos y sobre todo en quienes desempeñaron sucesivamente ambas ocupaciones. En estas actividades se estaría en contacto con contaminantes de posible acción cancerígena $a^{10,14,22}$.

Estes resultados, si bien avalan el riesgo ya indiscutido del tabaquismo referido al cáncer de pulmón, no coinciden totalmente con los obtenidos en otras poblaciones, lo que señala la necesidad de profundizar los estudios en nuestro medio a fin de clarificar la participación que distintos factores medioambientales y ocupacionales podrían tener en la determinación de los tipos histológicos, así como su relación sinergica o no con el hábito de fumar.

\section{Agradecimiento}

Al Dr. J.C. Morini, Director del Instituto de Inmunología por su lectura crítica del manuscrito.

MAHUAD, R. et al. Exposição ocupacional e câncer de pulmão em fumantes. Rev. Saíde Priblica, 28: 204-8, 1994. Foram estudados 211 pacientes do sexo masculino, fumantes, com câncer de pulmão, em Rosário (Argentina). Compararam-se as idades no momento do diagnóstico entre os tipos histológicos e ocupações e idade de início, duração e intensidade do hábito de fumar. A idade média dos pacientes diagnosticados foi de 61 anos, sem diferença por tipo histológico, porém por hábito de fumar: 58 anos os fumantes e 68 os ex-fumantes ( $p<0,0001$ ). Nesses pacientes encontrou-se predomínio de tumores epidermoides, o que poderia estar associado aos contaminantes ocupacionais presentes nesta fase de industrialização. A associação entre tipo celular e ocupação foi altamente significativa ( $\mathrm{p}<0,001$ ), encontrando-se o adenocarcinoma com maior frequiência nos trabalhadores administrativos e o epidermoide em outras ocupações, especialmente em homens que primeiramemte foram agricultores e depois metalúrgicos.

Descritores: Neoplasmas pulmonares, epidemiologia. Fumo, efeitos adversos. Ocupaçōes.

MAHUAD, R. et al. [Occupational exposure and lung cancer in smokers]. Rev, Saúde Pública, 28: 204-8, 1994. High male lung cancer incidence and mortality in Rosario city, Argentina, have been found in previous studies. A project was undertaken for the purpose of evaluating the life-time occupational history as well as the duration and intensity of cigarette smoking as 
determinants of histologic cell types in 211 male patients with primary lung cancer. Their histologic cell types were: squamous $39 \%$, adenocarcinoma $29 \%$, small cell $18 \%$, and others and not specified $14 \%$. An association was found between histologic cell types and occupations ( $\mathrm{p}<0.0001$ ), adenocarcinoma being more prevalent in office personnel, teachers, accountants, lawyers, and squamous in the other, supposedly dirtier working environments, mainly in those men who had begun to work in farming and later transferred to mechanics and metallurgy. These latter ones were diagnosed at a younger age than those in other occupations, with a significant difference for squamous and small cell. No differences in the smoking intensity were found between the occupational groups. The mean age these patients began to smoke at was 15 years for those with squamous and small cell, and 17 years for those with adenocarcinoma $(p<0.001)$. An interesting finding was the difference at their mean-age at diagnosis, 58 years for smokers and 68 for ex-smokers $(p<0.0001$ ). Studies are needed to elucidate the interplay of risk factors in the etiology of histologic subtypes of lung cancer.

Keywords: Lung neoplasms, epidemiology. Smoking, adverse effects. Occupations.

\section{Referenclas Bibliográficas}

1. AXELSON, $O$ et al. Lung cancer not attributable to smoking. Ann. N.Y.Acad.Sci, 609: 165-76, 1990.

2. AXELSON, O. \& STEENLAND, K. Indirect methods of assessing the effects of tobacco use in occupational studies. AmJIndMed., 13: 105-18, 1988.

3. BEARD, C. et al. Fifty-year trend in incidence rates of bronchogenic carcinoma by cell type in Olmsted County, Minnesota. J Natl.Cancer Inst., 80: 1404-10, 1988.

4. BRUMINI, R. ed. Cancer in Brazil: histopathological data. Rio de Janeiro, Ministry of Health, 1982.

5. BYERS, T. et al. Diet and lung cancer risk: findings from the Westem New York Diet Study, Am. J. Epidemiol., 125: 351-63, 1987.

6. CLASIFICACIÓN Intemacional Uniforme de Ocupaciones. Ginebra, Oficina Internacional del Trabajo, 1968.

7. FRANCO, E. L. et al. Correlation patterns of cancer relative frequencies with some socieconomic and demographic indicators in Brazil: an ecologic study. Int. J. Cancer, 41: 24-9, 1988.

8. KREYBERG, L. Histological lung cancertypes: a morphological and biological correlation. Acta Pathol. Microbiol. Scand. Suppl., (157): 1-92, 1962.

9. LEVI, E. et al. Socieconomic groups and cancer risk at death in the Swiss Canton of Vaud. Int. J. Epidemiol., 17: 711-7, 1988.

10. McDUFFIE, H. et al. Farming and exposure to chemicals in male lung cancer patients and their siblings. J.Occup.Med., 30: 55-9, 1988.

11. MIETTINEN, $O$ \& ROSSITER, C. Man-made mineral fibers and lung cancer. Scand.J. Work Environ. Health, 16: 221 31, 1990.

12. MORABIA, A. \& WYNDER, R. Cigarette smoking and lung cancer cell types. Cancer, $68: 2074-8,1991$.

13. MURATA, M. et al. A comparative epidemiologic study on geographic distributions of cancers of the lung and the large intestine in Japan.Jpn.J. CancerRes. (Gann), 79 :1005-16, 1988.

14. PASTORINO, V. et al. Proportion of lung cancer due to occupational exposure. Int. J. Cancer, 33: 231-7, 1984.

15. POLETTO, L. et al. Epidemiología del cancer en la ciudad de Rosario. Medicina (BsAs), 46 :35-42, 1986.

16. POLETTO, L. \& MORINI, J. Cancer mortality and some socioeconomic correlates in Rosario, Argentina. Cancer Lett., 49: 201-5, 1990.

17. POLETTO, L. et al. Prevalencia del hábito de fumar en jóvenes y sus padres: asociaciones relevantes con educación y ocupación. Rev. Saúde Pública, 25: 388-93, 1991.

18. PUFFER, R. \& GRIFFITH, G. Características de la mortalidad urbana. Washington, Organizacion Panamericana de la Salud, 1968.

19. ROSEN, M. et al. Changing smoking habits in Sweden: towards better health, but not for all. Int. J. Epidemiol., 19: 316-9, 1990.

20. SIMONATO, L. et al. Estimates of the proportion of lung cancer attributable to occupational exposure. Carcinogenesis, 9: 1159-63, 1988.

21. SMOKING and health: a report of the Surgeon General of the Human Services, US Department of Health and Human Services, Office on Smoking and Health. Washington, D.C. 1979.

22. STEHR-GREEN, P. Demographic and seasonal influences on human serum pesticide residue levels. J. Toxicol.Environ. Health, 27: 405-21, 1989.

23. TOMATIS, L. et al. Evaluation of the carcinogenicity of chemicals: a review of the monograph program of the International Agency for Research on Cancer. Cancer Res., 38: $877-85,1978$.

24. TOMATIS, L. et al. Human carcinogens so far identified. Jpn. J. Cancer Res., 80: 795-807, 1989.

25. VALAITIS, J. et al. Increasing incidence of adenocarcinoma of the lung. Cancer, 47: 1042-6, 1981.

26. VENA, J. et al. Air pollution as a risk factor in lung cancer. $A m$. J. Epidemiol., 116: 42-56, 1982.

27. VENA, J. et al. Occupation and lung cancer risk: an analysis by histologic subypes. Cancer, 56: 910-7, 1985.

28. VINEIS, P. \& SIMONATO, L. Proportion of lung and bladder cancers in males resulting from occupation: a systematic approach. Arch. Environ.Health, 46: 6-12, 1991.

29. WYNDER, E. et al. Ecologic study of lung cancer risk factors in the USA and Japan, with special reference to smoking and diet. Jpn.J. Cancer Res., 83: 418-23, 1992.

Recebido para publicação em 16.6.1993 Reapresentado em 17.3.1994 Aprovado para publicação em 24.3.1994 